\title{
Thermonuclear Plasma Steady States Generation
}

\author{
S. I. Fisenko, I. S. Fisenko, R. T. Rymkulov \\ Rusthermosynthesis JSC, Moscow, Russia \\ Email: StanislavFisenko@yandex.ru
}

Received November 2013

\begin{abstract}
This report is a systematic and complemented summary of the earlier published works by the authors [1-4]. The concept of gravitational radiation as a radiation of one level with the electromagnetic radiation is based on theoretically proved and experimentally confirmed fact of existence of electron's stationary states in own gravitational field, characterized by gravitational constant $\mathrm{K}=$ $10^{42}$ G (G-Newtonian gravitational constant) and by irremovable space-time curvature. The received results strictly correspond to principles of the relativistic theory of gravitation and the quantum mechanics. The given work contributes into further elaboration of the findings considering their application to dense high-temperature plasma of multiple-charge ions. This is due to quantitative character of electron gravitational radiation spectrum such that amplification of gravitational radiation may take place only in multiple-charge ion high-temperature plasma.
\end{abstract}

\section{Keywords}

Electron, Stationary States, Stable States, Pulse High-Current Discharge, Thermonuclear Fusion

\section{Introduction}

Last years' astronomical observations have brought to general relativity based astrophysics and cosmology such notions as "inflation", "dark matter", and "dark energy" thus urging to elaboration of the major number of recent alternatives to GR. New theories offer interpretation of these experimental data not invoking those notions for they seem to be wrong or artificial to the authors of these theories. The basic concept implies that gravity must agree with GR at least within Solar System at present epoch but may be essentially different on galaxy scale or higher as well as in early Universe. However all experimental attempts to detect gravitational radiation (based both on GR views and on alternative theories) yield no results. In elaboration of the relativistic theory of gravitation (namely the relativistic theory of gravitation rather than its particular case such as GR) the authors have obtained a model of gravitational interaction at quantum level having no equivalents and making gravitational radiation spectrum computing possible.

\section{Gravitational Radiation as a Radiation of the Same Level as Electromagnetic}

For a mathematical model of interest, which describes a banded spectrum of stationary states of electrons in the 
proper gravitational field, two aspects are of importance. First, in Einstein's field equations, $\kappa$ is a constant which relates the space-time geometrical properties with the distribution of physical matter, so that the origin of the equations is not connected with the numerical limitation of the $\kappa$ value. Only the requirement of conformity with the Newtonian Classical Theory of Gravity leads to the small value $\kappa=8 \pi G / c^{4}$, where G, c are, respectively, the Newtonian gravitational constant and the velocity of light. Such requirement follows from the primary concept of the Einstein General Theory of Relativity (GR) as a relativistic generalization of the Newtonian Theory of Gravity. Second. The most general form of relativistic gravitation equations are equations with the $\Lambda$ term. The limiting transition to weak fields leads to the equation

$$
\Delta \Phi=-4 \pi \rho G+\Lambda c^{2},
$$

where $\Phi$ is the field scalar potential, $\rho$ is the source density. This circumstance, eventually, is crucial for neglecting the $\Lambda$ term, because only in this case the GR is a generalization of the Classical Theory of Gravity. Therefore, the numerical values of $\kappa=8 \pi G / c^{4}$ and $\Lambda=0$ in the GR equations are not associated with the origin of the equations, but follow only from the conformity of the GR with the classical theory.

From the 70's onwards, it became obvious [5] that in the quantum region the numerical value of G is not compatible with the principles of quantum mechanics. The essence of the problem of the generalization of relativistic equations on the quantum level was thus outlined: such generalization must match the numerical values of the gravity constants in the quantum and classical regions.

In the development of these results, as a micro-level approximation of Einstein's field equations, a model is proposed, based on the following assumption [1,2]:

"The gravitational field within the region of localization of an elementary particle having a mass $\mathrm{m}_{0}$ is characterized by the values of the gravity constant $\mathrm{K}$ and of the constant $\Lambda$ that lead to the stationary states of the particle in its proper gravitational field, and the particle stationary states as such are the sources of the gravitational field with the Newtonian gravity constant G”.

The most general approach in the Gravity Theory is the one which takes twisting into account and treats the gravitational field as a gauge field, acting on equal terms with other fundamental fields ([6]).

Complexity of solving this problem compels us to employ a simpler approximation, namely: energy spectrum calculations in a relativistic fine-structure approximation. In this approximation the problem of the stationary states of an elementary source in the proper gravitational field will be reduced to solving the following equations:

$$
\begin{gathered}
f^{\prime \prime}+\left(\frac{v^{\prime}-\lambda^{\prime}}{2}+\frac{2}{r}\right) f^{\prime}+e^{\lambda}\left(K_{n}^{2} e^{-v}-K_{0}^{2}-\frac{l(l+1)}{r^{2}}\right) f=0 \\
-e^{-\lambda}\left(\frac{1}{r^{2}}-\frac{\lambda^{\prime}}{r}\right)+\frac{1}{r^{2}}+\Lambda=\beta(2 l+1)\left\{f^{2}\left[e^{-\lambda} K_{n}^{2}+K_{0}^{2}+\frac{l(l+1)}{r^{2}}\right]+f^{\prime 2} e^{-\lambda}\right\} \\
-e^{-\lambda}\left(\frac{1}{r^{2}}+\frac{v^{\prime}}{r}\right)+\frac{1}{r^{2}}+\Lambda=\beta(2 l+1)\left\{f^{2}\left[K_{0}^{2}-K_{n}^{2} e^{-v}+\frac{l(l+1)}{r^{2}}\right]-e^{\lambda} f^{\prime 2}\right\} \\
\left\{-\frac{1}{2}\left(v^{\prime \prime}+v^{\prime 2}\right)-\left(v^{\prime}+\lambda^{\prime}\right)\left(\frac{v^{\prime}}{4}+\frac{1}{r}\right)+\frac{1}{r^{2}}\left(1+e^{\lambda}\right)\right\}_{r=r_{n}}=0 \\
f\left(\sqrt{\Lambda^{-1}}\right)=0 \\
f\left(r_{n}\right)=0 \\
\lambda(0)=v(0)=0 \\
\int_{0}^{r_{n}} f^{2} r^{2} d r=1
\end{gathered}
$$

Equations (1)-(3) follow from Equations (9)-(10) 


$$
\begin{gathered}
\left\{-g^{\mu \nu} \frac{\partial}{\partial x_{\mu}} \frac{\partial}{\partial x_{v}}+g^{\mu \nu} \Gamma_{\mu \nu}^{\alpha} \frac{\partial}{\partial x_{\alpha}}-K_{0}^{2}\right\} \Psi=0 \\
R_{\mu \nu}-\frac{1}{2} g_{\mu \nu} R=-\kappa\left(T_{\mu \nu}-\mu g_{\mu v}\right),
\end{gathered}
$$

after the substitution of $\Psi$ in the form of $\Psi=f_{E l}(r) Y_{l m}(\theta, \varphi) \exp \left(\frac{-i E t}{\hbar}\right)$ into them and specific computations in the central-symmetry field metric with the interval defined by the expression [7]

$$
d S^{2}=c^{2} e^{v} d t^{2}-r^{2}\left(d \theta^{2}+\sin ^{2} \theta d \varphi^{2}\right)-e^{\lambda} d r^{2}
$$

The following notation is used above: $f_{E l}$ is the radial wave function that describes the states with a definite energy $\mathrm{E}$ and the orbital moment $\mathrm{l}$ (hereafter the subscripts $\mathrm{El}$ are omitted), $Y_{l m}(\theta, \varphi)$-are spherical functions, $K_{n}=E_{n} / \hbar c, \quad K_{0}=c m_{0} / \hbar, \quad \beta=(\kappa / 4 \pi)\left(\hbar / m_{0}\right)$.

Condition (4) defines $r_{n}$, whereas Equations (10) through (7) are the boundary conditions and the normalization condition for the function $f$, respectively. Condition (4) in the general case has the form $R\left(K, r_{n}\right)=R\left(G, r_{n}\right)$. Neglecting the proper gravitational field with the constant $\mathrm{G}$, we shall write down this condition as $R\left(K, r_{n}\right)=0$, to which Equality (4) actually corresponds.

The right-hand sides of Equations (2)-(3) are calculated basing on the general expression for the energy-momentum tensor of the complex scalar field:

$$
T_{\mu \nu}=\Psi_{, \mu}^{+} \Psi_{, \nu}+\Psi_{, \nu}^{+} \Psi_{, \mu}-\left(\Psi_{, \mu}^{+} \Psi^{\mu}-K_{0}^{2} \Psi^{+} \Psi\right)
$$

The appropriate components $\mathrm{T}_{\mu v}$ are obtained by summation over the index $\mathrm{T}$ with application of characteristic identities for spherical functions [8] after the substitution of $\Psi=f(r) Y_{l m}(\theta, \varphi) \exp \left(\frac{-i E t}{\hbar}\right)$ into (12).

In its simplest approximation (from the point of view of the original mathematical estimates) the problem on steady states in proper gravitational field (with constants $\mathrm{K}$ and $\Lambda$ ) is solved by [1]. The solution of this problem provides the following conclusions.

1) With the numeric values $\mathrm{K} \approx 5.1 \times 10^{31} \mathrm{~N} \cdot \mathrm{m}^{2} \cdot \mathrm{kg}^{-2}$ and $\Lambda=4.4 \times 10^{29} \mathrm{~m}^{-2}$ there is a spectrum of steady states of the electron in proper gravitational field $(0.511 \mathrm{MeV} \ldots 0.681 \mathrm{MeV})$. The basic state is the observed electron rest energy $0.511 \mathrm{MeV}$.

2) These steady states are the sources of the gravitational field with the G constant.

3) The transitions to stationary states of the electron in proper gravitational field cause gravitational emission, which is characterized by constant $\mathrm{K}$, i.e. gravitational emission is an emission of the same level as electromagnetic (electric charge e, gravitational charge $m \sqrt{K}$ ). In this respect there is no point in saying that gravitational effects in the quantum area are characterized by the $\mathrm{G}$ constant, as this constant belongs only to the macroscopic area and cannot be transferred to the quantum level (which is also evident from the negative results of registration of gravitational waves with the $\mathrm{G}$ constant, they do not exist).

Existence of such numerical value $\Lambda$ denotes a phenomenon having a deep physical sense: introduction into density of the Lagrange function of a constant member independent on a state of the field. This means that the time-space has an inherent curving which is connected with neither the matter nor the gravitational waves. The distance at which the gravitational field with the constant $\mathrm{K}$ is localized is less than the Compton wavelength, and for the electron, for example, this value is of the order of its classical radius. At distances larger than this one, the gravitational field is characterized by the constant $\mathrm{G}$, i.e., correct transition to Classical GR holds.

There is certain analytic interest in $\beta$-decay processes with asymmetry of emitted electrons [9], due to (as it is supposed to be) parity violation in weak interactions. $\beta$-asymmetry in angular distribution of electrons was registered for the first time during experiments with polarized nucleuses ${ }_{27} \mathrm{Co}^{60}, \beta$-spectrum of which is characterized by energies of $\mathrm{MeV}$. If in the process of $\beta$-decay exited electrons are born, then along with decay scheme

$$
n \rightarrow p+e^{-}+\tilde{v}
$$

there will be also decay scheme 


$$
n \rightarrow p+\left(e^{*}\right)^{-}+\tilde{v} \rightarrow e^{-}+\tilde{\gamma}+\tilde{v}
$$

where $\tilde{\gamma}$ is a graviton.

Decay (14) is energetically limited by energy values of $1 \mathrm{MeV}$ order (in rough approximation), taking into consideration that the difference between lower excitation level of electron's energy (in own gravitational field) and general $<100 \mathrm{keV}$ and the very character $\beta$-spectrum. Consequently, ${ }_{27} \mathrm{Co}^{60}$ nucleuses decay can proceed with equal probability as it is described in scheme (13) or in scheme (14). For the light nucleuses, such as ${ }_{1} \mathrm{H}^{3}$ $\beta$-decay can only proceed as it is described in scheme (13). At the same time, emission of graviton by electron in magnetic field can be exactly the reason for $\beta$-asymmetry in angular distribution of electrons. If so, then the phenomenon of $\beta$-asymmetry will not be observed in light $\beta$-radioactive nucleuses. This would mean that $\beta$-asymmetry in angular distribution of electrons, which is interpreted as parity violation, is the result of electron's gravitational emission, which should be manifested in existence of lower border $\beta$-decay, as that's where $\beta$-asymmetry appears to be.

Using Kerr-Newman metric for estimation of the numerical value of K one can obtain the formula [7]

$$
K=\frac{r^{2}}{\left(m c r^{2} / L-L / m c\right)\left(m / r c^{2}-e^{2} / r^{2} c^{4}\right)} ;
$$

where $r, m, e, L$ are classical electron radius, mass, charge, orbital momentum respectively, and c is the speed of light.

Despite the fact that we used external metric and orbital momentum in deriving the formula (15), its use is legitimate for the orbital momentum of a particle in internal metric equal to the electron spin by an order of magnitude. The estimation of $\mathrm{K}$ from the formula (15) using the numerical values of the abovementioned arguments agrees with the estimation that stands in correspondence with numerical values of electron energy spectrum in proper gravitational field. This may suggest that the physical nature of spin is possibly such that these are just values of the orbital momentum of a particle in proper gravitational field.

\section{Spectral Lines Widening of the Radiation of Multiple-Charge Ions}

Figures 1 and 2 show characteristic parts of micropinch soft X-ray radiation spectrum. Micropinch spectrum line widening does not correspond to existing electromagnetic conceptions but corresponds to such plasma thermo-dynamic states which can only be obtained with the help of compression by gravitational field [2,3], radiation flashes of which takes place during plasma thermalization in a discharge local space. Such statement is based on the comparison of experimental and expected parts of the spectrum shown in Figure 2(a) and (b). Adjustment of the expected spectrum portion to the experimental one (see [1]) was made by selecting average values of density $\rho$, electron temperature Te and velocity gradient $U$ of the substance hydrodynamic motion.

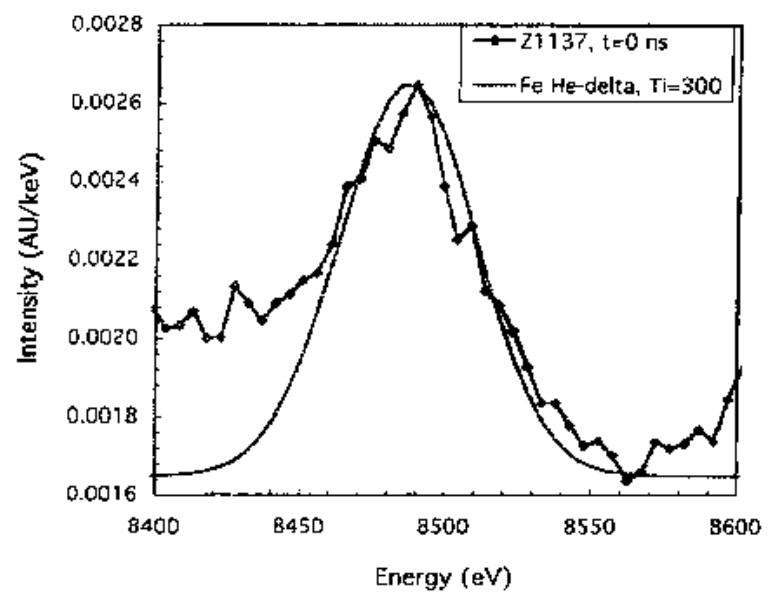

Figure 1. Measured Fe He- $\delta$ line at $8.488 \mathrm{keV}$ (broken curve) compared to calculation (smooth curve) [10]. 

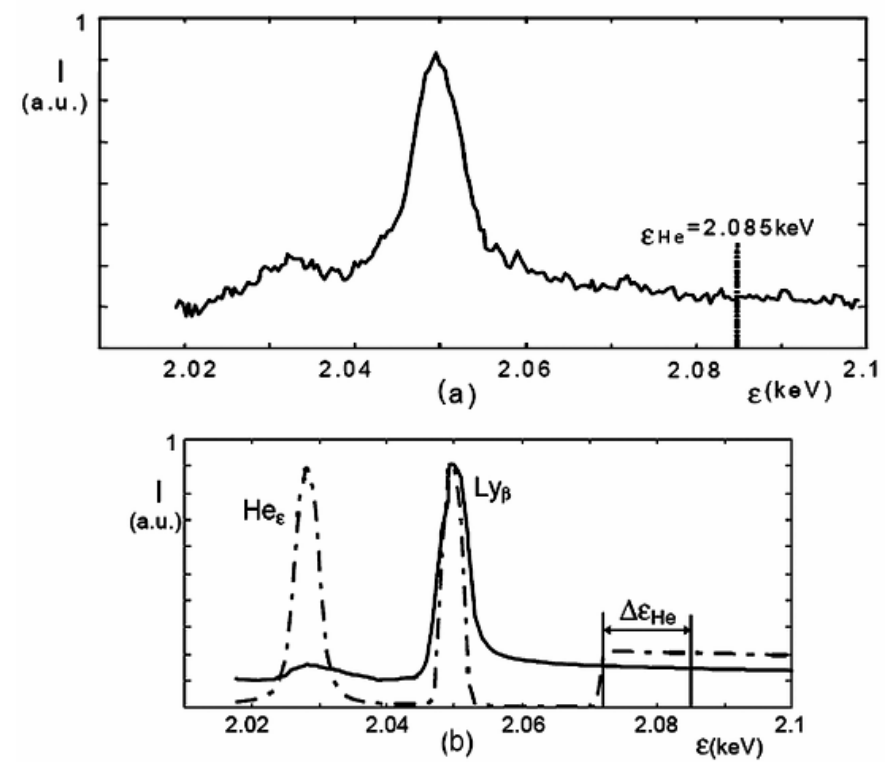

Figure 2. Experimental (a) and calculated (b) parts of a micropinch spectrum normalized for line Ly $\beta$ intensity in the area of the basic state ionization threshold of He-like ions. The firm line in variant (b) corresponds to density of $0.1 \mathrm{~g} / \mathrm{cm}^{3}$, the dotted line- to $0.01 \mathrm{~g} / \mathrm{cm}^{3}$; it was assumed that $\mathrm{T}_{\mathrm{e}}=0.35 \mathrm{keV}$, (see [1]).

As a mechanism of spectrum lines widening, a Doppler, radiation and impact widening were considered. Such adjustment according to said widening mechanisms does not lead to complete reproduction of the registered part of the micropinch radiation spectrum. This is the evidence (under the condition of independent conformation of the macroscopic parameters adjustment) of additional widening mechanism existence due to electron excited states and corresponding gravitational radiation spectrum part already not having clearly expressed lines because of energy transfer in the spectrum to the long-wave area.

That is to say that the additional mechanism of spectral lines widening of the characteristic electromagnetic radiation of multiple-charge ions (in the conditions of plasma compression by radiated gravitational field) is the only and unequivocal way of quenching electrons excited states at the radiating energy levels of ions and exciting these levels by gravitational radiation at resonance frequencies. Such increase in probability of ion transitions in other states results in additional spectral lines widening of the characteristic radiation. The reason for quick degradation of micropinches in various pulse high-currency discharges with multiple-charge ions is also clear. There is only partial thermolization of accelerated plasma with the power of gravitational radiation not sufficient for maintaining steady states [4].

\section{Direct Experimental Measurements}

\subsection{Emission Spectra of Electron Beams Interacting with Cathode}

Technically, the most accessible is the use of electron beams, taking into account the above made reservation (narrow width of the energy levels of stationary states of the beam's electrons in its own gravitational field). In rough approximation, to estimate the numerical values of the stationary states energies in its own gravitational field, the following values were obtained:

$$
E_{1}=0.511 \mathrm{MeV}, E_{2}=0.638 \mathrm{MeV}, \ldots . . E_{\infty}=0.681 \mathrm{MeV} .
$$

Certainly, numerical values of the main stationary state $E_{1}=0.11 \mathrm{MeV}$ are exact, as well as the energy range of stationary states itself, equal to $171 \mathrm{keV}$, that is, as we approach the $E_{\infty}$, numerical values of spectrum energies are evaluated more accurately. The largest error occurs in the evaluation of the numerical value of the first stationary state (and, surely, of the next few states); after that, an error decreases as we approach the $E_{\infty}$. The idea of the experiment is that during deceleration of the electron beam on target, along with the well known 
and well studied continuous spectrum of the deceleration electromagnetic emission and linear spectrum of characteristic radiation, a gravitational emission of a linear spectrum will take place. Because of the impact of emission, the linear spectrum of gravitational emission energies will be offset relative to transition energies $\left(E_{2}-E_{1}\right),\left(E_{3}-E_{2}\right)$, etc. The magnitude of this offset $\Delta E$ for electrons with good accuracy is given by the expression:

$$
\Delta E \cong 0.98 E^{2}[\mathrm{MeV}]
$$

It follows from (16), that the energies of the gravitational emission spectral lines are offset relative to the transition energies for about 6 to $30 \mathrm{keV}$ (again, with good accuracy for top energy levels of an electron in its own gravitational field). Therefore, the gravitational emission linear spectrum is guaranteed to stay in an energy range of $70 \mathrm{keV}$ to $140 \mathrm{keV}$ (perhaps, even shifted down by energy value). Unlike the well known methods of recording electromagnetic emission (throughout all its range), no such methods of recording exist for gravitational emission. For linear spectrum of gravitational emission the above-mentioned feature (lineation of spectrum) can be used, compared to continuous spectrum of the deceleration electromagnetic emission (in matching by energies ranges). While doing so, of course, imposition of lines of characteristic electromagnetic emission on continuous spectrum of deceleration emission should be taken into consideration (taking into account the anode specific material).

The action of the gravitational quantum on the detector, in terms of energy, in principle, is similar to action of the photon. The main problem will be the resolution of the detector, taking into account narrow width of the gravitational emission lines. That's the fact with which will be connected the main difficulty of spectrum lines recording in the wavelength range of (0.01 - 0.04) $\AA$ (corresponding to electron gravitational emission spectrum). Gravitational emission spectrum lines will be recorded either in form of narrow maximums (similar to characteristic emission lines), or in form of minimums, depending on resolution of the detector. It is also clear that the measurements should be conducted at various values of the accelerating potential of electrons, in any case providing for emission range of $(0.01-0.04) \AA$. Again, known character of the characteristic emission spectra of the used anode metals will allow avoiding possible errors in identification of the gravitational emission spectrum lines.

\subsection{Emission Spectra of Electron Beams on Foil for Various Materials and Their Energy Spectra after Passing the Foil}

This data, of course, should be completed both by electron gravitational emission lines and by stationary states energy spectrum of an electron in its own gravitational field. Previously, the following measurements were carried out. Figure 3 shows the energy spectra of electron beams in a pulsed accelerator, measured with a semicircular magnetic spectrometer. Presence of two peaks on energy spectra is associated with an operating mode feature of the pulsed electron accelerator, i.e., the presence of the secondary peak of lower power. This leads to the second (low-energy) peak in energy spectral distribution. Measurement error in the middle and soft part of the spectrum does not exceed $\pm 2 \%$. The energy spectrum of electrons passing through the anode mesh, and spectra of electrons passing through the foil attached above the mesh anode of the accelerator were measured with a magnetic spectrometer. This data, as well as a calculated range, are shown in Figure 3. Similar measurements were taken for Ti foil (foil thickness was 50 microns) and Ta foil (of 10 microns thickness). For Ti and Ta upper limits of measurements were $0.148 \mathrm{MeV}$ and $0.168 \mathrm{MeV}$ correspondingly; above these limits the measurement errors substantially increase (for the accelerator used). Figur $\mathbf{4}$ shows the difference of normalized spectral densities of the theoretical and experimental spectra of electrons after passing through the $\mathrm{Ti}$, Ta and $\mathrm{Al}$ foils. These data indicate the presence spectrum of energetic states of electrons in their own gravitational field, which are excited while passing through foil.

$$
\begin{gathered}
\frac{\Delta N_{e}}{\Delta E}, \frac{\text { electron }}{\mathrm{MeV}} \\
\delta\left(\frac{\Delta N_{e}}{\Delta E}\right), \frac{\text { electron }}{\mathrm{MeV}}
\end{gathered}
$$

Emission spectra in this series of experiments were not measured, and the measurements should be performed with detectors of higher sensitivity with simultaneous measuring of emission spectra. Provided data is not suffi- 


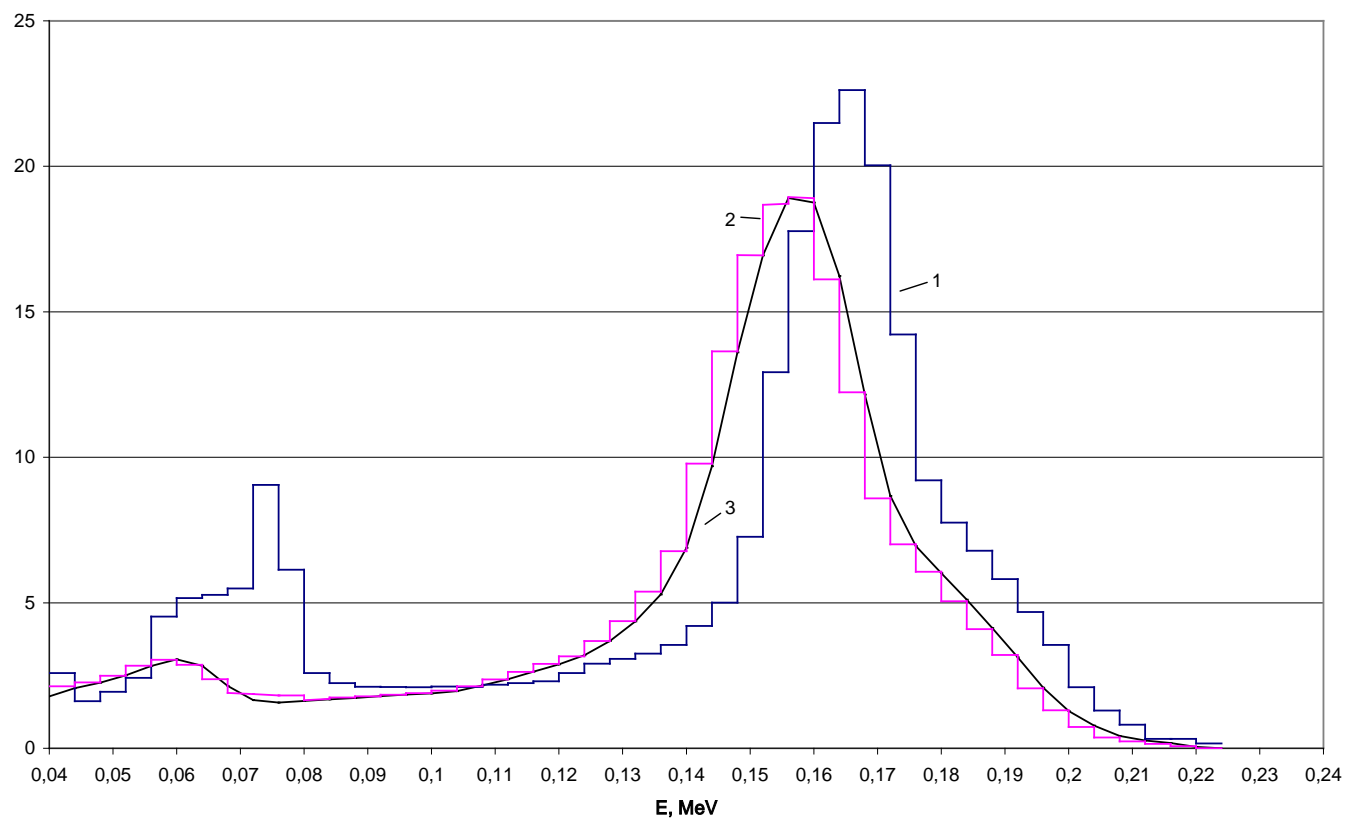

Figure 3. Electron energy spectra: 1 -after passing the grid, 2-after passing the Al foil $13 \mu \mathrm{m}$ thick; 3 spectrum calculation according to ELIZA program based on the database [11] for each spectrum 1 . The spectrum is normalized by the standard.

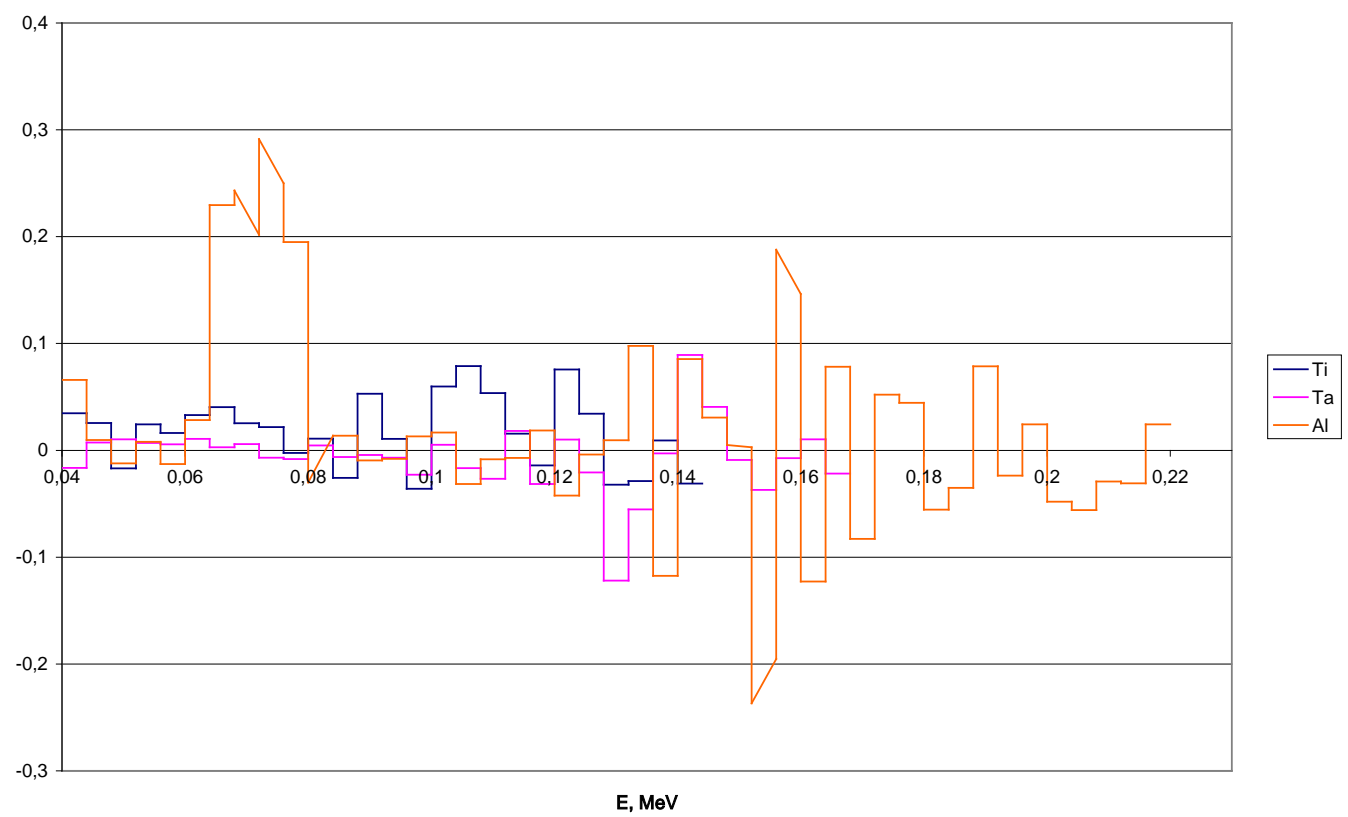

Figure 4. Difference of spectral density for theoretical and experimental spectra of electrons passed through $\mathrm{Ti}$, Ta and $\mathrm{Al}$ foils.

cient for numerical identification of the energy state spectrum of the electrons in their own gravitational field, but the very existence of the spectrum, according to this data, is unquestionable.

\section{Thermonuclear Plasma Steady States Generation}

The problem of controlled fusion realization is directly connected to obtaining steady state of dense high-temperature plasma. 
It can also be unambiguously stated that the present state of the art (retaining plasma by magnetic fields of various configurations, squeezing by laser radiation) does not solve the problem of dense high-temperature plasma retention for a time required for the reaction of nuclear fusion but only solving the problem of heating plasma to the state when these reactions can exist. In the offered method of forming dense-high temperature plasma steady states for nuclear fusion a new fundamental concept is used, namely retaining plasma by radiated gravitational field as radiation of the same kind as electromagnetic:

Forming and accelerating binary plasma with multivalent ions by accelerating magnetic field in a pulse highcurrent discharge.

Injection of binary plasma from the space of the accelerating magnetic field:

exciting stationary states of an electron in its own gravitational field in the range of energy up to $171 \mathrm{keV}$ with following radiation under the condition of quenching lower excited energy levels of ion electron shell of a heavy component (including quenching excited state of electrons directly in nuclei of small sequential number as carbon) when retarding plasma bunch ejected from the space of the accelerating magnetic field. Cascade transitions from the upper levels are realized in the process of gravitational radiation energy transit to long-wave range.

The sequence of the operations is carried out in a two-sectional chamber of MAGO installation (Figure 5, developed in Experimental Physics Research Institute, Sarov, [12]; the structure of the installation is most suitable for the claimed method of forming steady states of the dense high-temperature plasma) with magnetodynamic outflow of plasma and further conversion of the plasma bunch energy (in the process of quenching) in the plasma heat energy for securing both further plasma heating and exciting gravitational radiation and its transit into a long-wave part of the spectrum with consequent plasma compression in the condition of radiation blocking and increasing [4].

Of interest, there are two modes of the installation operations depending on the work gas composition [13].

1) A composition with hydrogen and (for example) xenon providing for achieving steady states of plasma with consequent realization of thermonuclear reactions for compositions of $(\mathrm{d}+\mathrm{t})+$ multi-charge atoms type.

Fusion reaction creating helium generates neutrons

$$
{ }_{1}^{2} \mathrm{H}+{ }_{1}^{3} \mathrm{H} \rightarrow{ }_{2}^{4} \mathrm{He}+{ }_{0}^{1} n+17.6 \mathrm{MeV}
$$

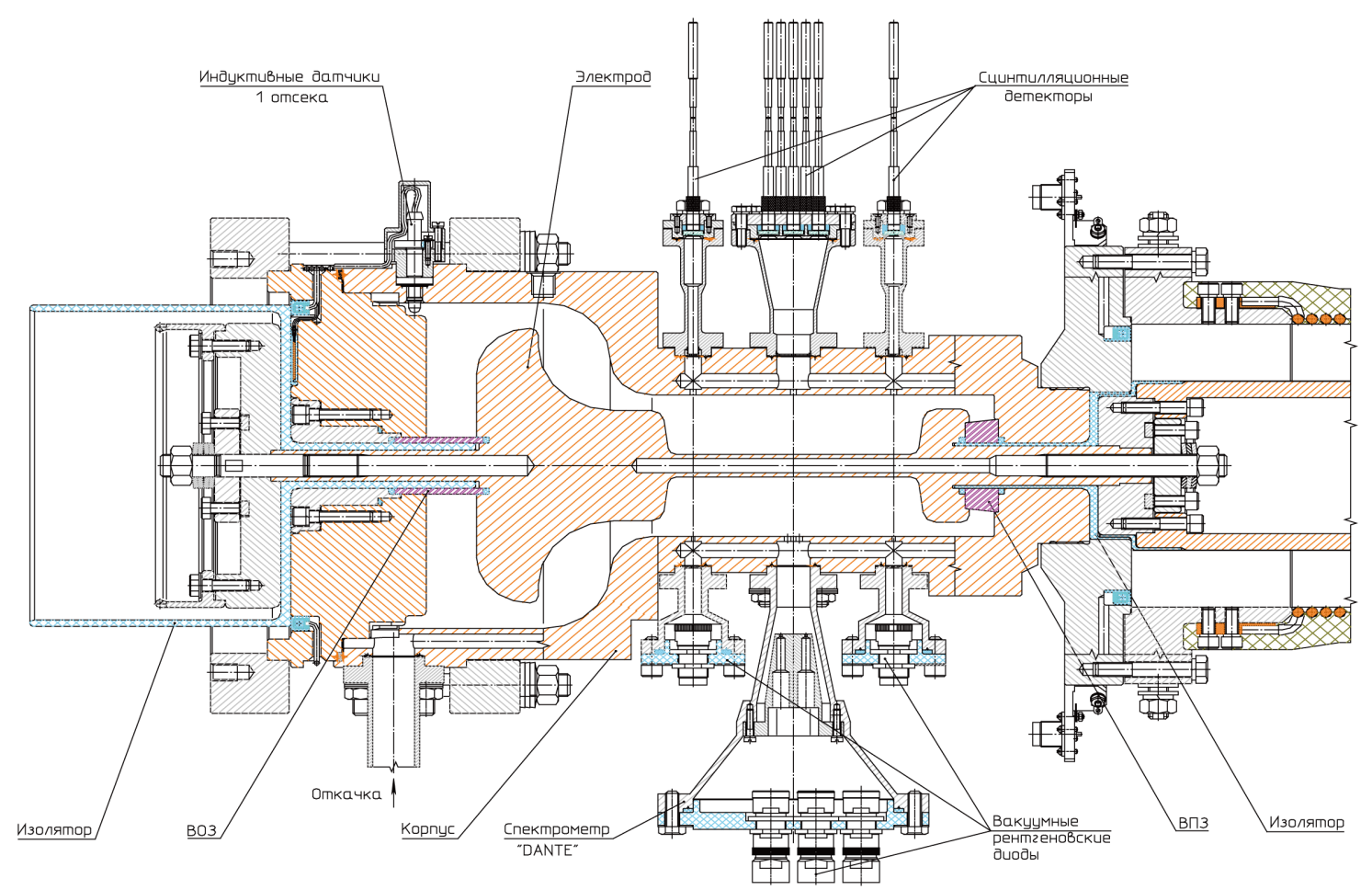

Figure 5. Outline drawing of the discharge chamber (MAGO chamber) and X-ray diagnostic system. 
and was embodied in the well-known Teller-Ulam design with radiation implosion.

An application of the compression-by-the-radiated-gravitational-field design, unlike the Teller-Ulam design, is not limited by the minimum discharge power attendant to the usage of a plutonium nucleus. This means both a feasibility of this fast fusion reaction a steady mode for low-power discharges, and (under certain conditions) a feasibility of explosive energy release without the use of fissile elements (like plutonium and uranium) for highpower discharges.

2) A composition with hydrogen and carbon providing thermonuclear reactions of carbon cycle in plasma steady state mode, including energy pick-up in the form of electromagnetic radiation energy

$\mathrm{CNO}$ cycle is a set of fusion reactions resulting in conversion of hydrogen into helium using carbon as a catalyst.

In a compact notation, this cycle would be written as

$$
{ }^{12} \mathrm{C}(\mathrm{p}, \gamma){ }^{13} \mathrm{~N}\left(\mathrm{e}^{+} v\right){ }^{13} \mathrm{C}(\mathrm{p}, \gamma){ }^{14} \mathrm{~N}(\mathrm{p}, \gamma){ }^{15} \mathrm{O}\left(\mathrm{e}^{+} v\right){ }^{15} \mathrm{~N}(\mathrm{p}, \alpha){ }^{12} \mathrm{C}
$$

The ${ }^{15} \mathrm{~N}(\mathrm{p}, \alpha){ }^{12} \mathrm{C}$ reaction rounds the cycle out. The net result is that four protons turn into an $\alpha$-particle${ }^{4} \mathrm{He}$ nucleus with no neutrons among end products of the cycle. Producing one helium nucleus releases $25 \mathrm{MeV}$, the produced neutrinos carry away about $5 \%$ more of that energy. A peculiarity of such fusion reaction cycle is that it occurs in natural conditions of astrophysical objects. At the same time, e.g. the design features of MAGO facility allow achieving such a volt-ampere characteristics (VAC) mode that boosts the speed of carbon cycle reactions. An implementation of carbon cycle in the gravitational compression design may possibly become a basic design to form steady states of the thermonuclear plasma, bearing in mind low abundance of hydrogen and lithium isotopes that react with no neutrons being produced either.

\section{References}

[1] Fisenko, S.I. and Fisenko, I.S. (2009) Gravitational Interaction on Quantum Level and Consequences Thereof. The Old and New Concepts of Physics, 6, 495-521.

[2] Fisenko, S.I. and Fisenko, I.S. (2011) Gravitational Radiation of the Relativistic Theory of Gravitation. British Journal of Science, 2, 1-20.

[3] Fisenko, S.I., Fisenko, I.S. (2012) The Discrete Energy Spectrum of the Gravitational Radiation in the Relativistic Theory of Gravitation. International Journal of Theoretical and Applied Physics (IJTAP), 2, 32-39.

[4] Fisenko, S.I. and Fisenko, I.S. (2013) Method of Forming Stable States of Dense High-Temperature Plasma. Journal of Modern Physics, 4, 481-485.

[5] Siravam, C. and Sinha, K. (1979) The Concept of “Strong” Gravity. Physics Reports, 51, 112-123.

[6] Ivanenko, D.D., et al. (1985) Gauge Theory of Gravity. MGU Publishing House, Moscow, 71-73.

[7] Landau, L.D. and Lifshitz, E.M. (1976) Field Theory. Publishing House “Nauka”, Moscow, 451-457.

[8] Warshalovich, D.A., et al. (1975) Quantum Theory of Angular Momentum. Publishing House “Nauka”, Leningrad, 282-285.

[9] Wu, Z.S. and Moshkovsky, S.A. (1970) $\beta$-Decay. Atomizdat, Moscow, 121-135.

[10] Haines, M.G., et al. (2006) Viscous Heating at Stagnation in Z-Pinches. PRL, 96, 075003-075008.

[11] Cullen, D.E., et al., (1994) Report IAEA—NDS-158.

[12] Burenkov, O.M., Dolin, Y.N., Duday, P.V., Dudin, V.I., Ivanov, V.A., Ivanovsky, A.V., Karpov, G.V., et al. (2012) New Configuration of Experiments for MAGO Program, XIV International Conference on Megagauss Magnetic Field Generation and Related Topics, October 14-19, 2012, Maui, Havaii, 95-99.

[13] Fissenko, S. and Fissenko, I. (2005) (IPN WO2005/109970 A1): Method of Forming Stable States of Dense HighTemperfature Plasma. PCT Gazette No. 46, 553. 